\title{
New Synthesis: Exploring the Chemical Links in Ecological Food Webs
}

\author{
R. A. Raguso
}

Published online: 5 May 2012

(C) Springer Science+Business Media, LLC 2012

For the past year, contributors to New Synthesis have explored conceptual and technological frontiers that promise to stretch the field of Chemical Ecology in new directions. One such opportunity is the integration of chemical signals and chemoreception into community ecology. Studies of "food web" networks have revealed the disproportionate importance of "keystone" species, the resiliency of certain interactions (e.g., generalized herbivores or pollinators with their hosts) to species turnover or disturbance, and the context dependence of network structure, with direct impacts on resource management and conservation as well as ecological theory.

At first glance, there would appear to be little common ground between chemical and community ecology, as scientists in these fields tend to employ different methodological approaches to their craft. For example, the chemical, physiological and behavioral assays used to study the pollination of sexually deceptive orchids by bees usually are not utilized by teams of ecologists measuring links between the same bee species and tens of other flowering plant species that they visit for nectar or pollen. Indeed, the null models used in some food web studies begin with the assumption that all species are equally likely to interact. Though heuristically useful, this assumption strikes many chemical ecologists and behaviorists as unreasonable. When "links" are found to be stronger or weaker than null expectations, biological explanations are sought. Until recently, community ecologists have been more likely to invoke habitat use, phenology, or population density than specific phenotypic or behavioral traits to explain how food webs are constructed.

How might chemistry explain link strength - or its absence - in ecological networks? Three recent or ongoing studies illustrate the dialectical potential between chemical ecology and community ecology. At the simplest (agricultural plot) scale, Poelman et al. (2009) have documented the effects of compositional variation in plant resistance compounds (glucosinolates) on herbivore diversity and abundance, using different cultivars of Brassica oleracea and wild B. nigra mustard plants in The Netherlands. Total glucosinolate concentration has no effect on insect diversity indices, whereas cultivars high in glucoiberin content but low in glucoraphanin host fewer aphids and caterpillars. Subsequent bioassays revealed at least two non-competitive mechanisms for this result: low oviposition and poor larval performance on high-glucoiberin cultivars by specialist (Pieris rapae) and generalist (Mamestra brassicae) lepidopterans. Additional work by the Wageningen group has revealed the bottom-up effects of specific glucosinolates on higher trophic levels in this agroecosystem network, including parasitoid and hyperparasitoid wasps. Thus, leaf

\footnotetext{
R. A. Raguso $(\bowtie)$

Cornell University,

Ithaca, NY 14853, USA

e-mail: rar229@cornell.edu
}

chemistry has strong predictive power in the food web that stretches across a field of Brussels sprouts.

At a more complex (floral marketplace) scale, Junker and colleagues (2010) studied a network of 35 flowering plant species and 164 flowervisiting insect species in a German meadow. Most observed links deviated significantly from null model expectations, either more frequently ("hot links") or less frequently ("cold links") than expected, and several of these links were investigated using behavioral choice assays, through the use of a field-portable olfactometer. Interestingly, hot links between specific pollinators and flowers usually coincided with significant olfactory preference (either innate or learned) for that flower's scent, and the few testable cold links were reinforced by actual repellence, rather than indifference. This is the first community-level demonstration that floral volatile chemistry underlies both positive and negative network links, as an ecological filter.

At the most complex (landscape-ecosystem) scale, Zimmer and Ferrer (2007) suggest that toxins of microbial origin can propagate through food webs due to their powerful effects on predation and energy flow, and might profitably be considered "keystone molecules". Tetrodotoxin (TTX) and saxitoxin (STX) are potent neurotoxins that block sodium channels, often resulting in paralysis or death. Marine, riparian, and some terrestrial communities are heavily structured by the uptake and use of TTX as a defense compound (e.g., by newts), and the degree to which their predators (e.g., garter snakes) can tolerate such compounds. Similarly, marine food webs structured around kelp beds are strongly impacted by sea otter predation on urchins or butter clams, a decision that is directly impacted by the presence of STX in clams (and ultimately, on dinoflagellates as the source of STX).

Thus, diverse organic compounds ranging from simple ubiquitous volatiles (e.g., monoterpenes) to more complex defense compounds (glucosinolates and herocyclic alkaloids) do more than mediate the binary organismal interactions studied by chemical ecologists in the first half century of our field's history. They lend structure to the food webs through which abiotic sources of energy enter and flow through the biosphere.

\section{References}

Poelman, E. H., van Dam, N. M., van Loon, J. J. A., Vet, L. E. M., and Dicke, M. 2009. Chemical diversity in Brassica oleracea affects biodiversity of insect herbivores. Ecology 90:1863-1877.

JuNKER, R. R., HöCHERL, N., and BLÜTHGEN, N. 2010. Responses to olfactory signals reflect network structure of flower-visitor interactions. Journal of Animal Ecology 79:818-823.

ZIMMER, R. K. and FERRER, R. P. 2007. Neuroecology, chemical defense and the keystone species concept. Biological Bulletin 213:208-225. 